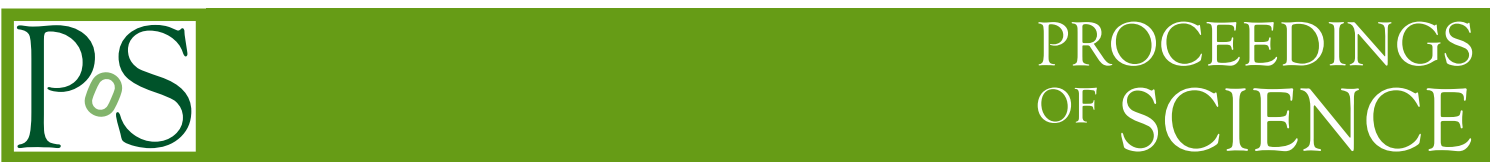

\title{
First results of the R2D2 project
}

\author{
Anselmo Meregaglia ${ }^{a, *}$ \\ ${ }^{a} C E N B G$, Université de Bordeaux, CNRS/IN2P3, 33175 Gradignan, France \\ E-mail: amerega@in2p3.fr
}

The search for neutrinoless double beta decay $(0 v \beta \beta)$ could cast light on one critical piece missing in our knowledge i.e. the nature of the neutrino mass. Its observation is indeed the most sensitive experimental way to prove that neutrino is a Majorana particle. The observation of such a potentially rare process demands a detector with an excellent energy resolution, an extremely low radioactivity and a large mass of emitter isotope. Nowadays many techniques are pursued but none of them meets all the requirements at the same time. The goal of R2D2 is to prove that a spherical high pressure gas TPC filled with xenon could meet all the requirements and provide an ideal detector for the $0 v \beta \beta$ decay search. The first prototype has demonstrated an excellent resolution with argon meeting the expectations and independently on the particle track's length. In the presented talk the first R2D2 results are discussed as well as the project roadmap and future developments.

40th International Conference on High Energy physics - ICHEP2020

July 28 - August 6, 2020

Prague, Czech Republic (virtual meeting)

\footnotetext{
${ }^{*}$ Speaker
} 


\section{Introduction}

To assess if the neutrino is a Dirac or a Majorana particle is a very important question in the panorama of neutrino physics, and most sensitive experimental way to address it is an observation of the so called neutrinoless double beta decay $(0 v \beta \beta)$. The measurement of the $0 v \beta \beta$ relies on the observation of a peak in the distribution of the energy of the two electrons corresponding to the $Q_{\beta \beta}$ of the reaction.

The main requirements to search for such a rare phenomenon are:

- Excellent energy resolution. This is critical in order to have a narrow peak of the $0 v \beta \beta$ signal and minimize the background coming from the $2 v \beta \beta$ continuous spectrum. Furthermore a good energy resolution would allow to reduce the width of the region of interest (ROI) reducing therefore the external background.

- Low background detector. This will reduce the number of events in the ROI, and the most natural way to meet such a requirement is to have a low material budget detector.

- Large isotope masses. Ongoing experiments are just hitting the inverted mass hierarchy region whereas to fully cover it and reach a limit on the effective mass probed $\mathrm{m}_{e e}$ of few $\mathrm{meV}$, a ton scale experiment is needed.

The goal of the R2D2 (Rare Decays with Radial Detector) project is to develop a detector meeting all the requirements at the same time. R2D2 is indeed an R\&D program aiming at the development of a zero background ton scale detector to search for the neutrinoless double beta decay. The proposed technology relies on the use of a spherical high pressure xenon time projection chamber (TPC).

The first important target of the proposed R\&D is to demonstrate that a resolution at the level of $1 \%$ can be achieved at the ${ }^{136} \mathrm{Xe} Q_{\beta \beta}$ of $2.458 \mathrm{MeV}$.

\section{Detector}

The R2D2 detector is a spherical high pressure xenon TPC based on the developments carried out for the search of dark matter within the NEWS-G collaboration [1]. The working principle is shown in Fig. 1: a central anode at high voltage collects and amplifies the electrons produced by the ionisation of the passage of particles through the xenon gas.

The proposed detector has key features very important in the search of rare phenomena such as $0 v \beta \beta$.

- High energy resolution.

- Extremely low (potentially zero) background due to the minimal material budget.

- Scalability to large isotope masses ( 1 ton $=1 \mathrm{~m}$ radius at 40 bars).

- Low detection threshold at the level of $30 \mathrm{eV}$ i.e. single electron signal.

- High detection efficiency ( 65\% after selection cuts). 


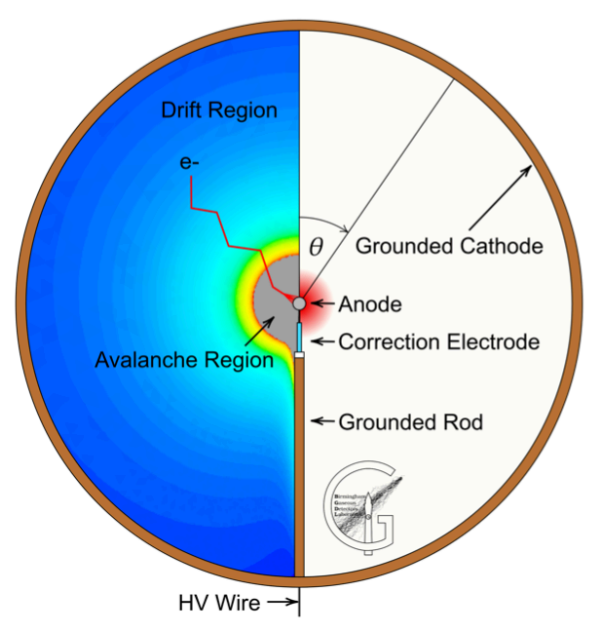

Figure 1: Working principle of the spherical TPC detector.

- Simplicity of the detector readout with only one (or few in the upgraded version) readout channels.

A dedicated simulation was developed to assess the detector sensitivity [2]: with $50 \mathrm{~kg}$ xenon at 40 bars in a sphere of $37 \mathrm{~cm}$ radius, in one year a limit on the $0 v \beta \beta$ half life of $2.5 \times 10^{25}$ years can be obtained, corresponding to an effective mass $m_{e e}$ smaller than $160-330 \mathrm{meV}$ depending on the matrix element values. The estimated efficiency is at the level of $64 \%$ and the expected number of background events in the ROI is 2 events per year. The published results depend on the assumption made, the most stringent one being an energy resolution of $1 \%$ FWHM at the $Q_{\beta \beta}$ of $2.458 \mathrm{MeV}$. The goal of the R2D2 R\&D is indeed to demonstrate that the assumptions are correct to validate the computed detector sensitivity.

\section{Experimental setup}

The R\&D was funded by IN2P3 in 2018 with the main goal of demonstration that the desired energy resolution is achievable. A $20 \mathrm{~cm}$ radius aluminium sphere was conceived and built at CENBG mechanical workshop (see Fig. 2(a) and Fig. 2(b)). Such a sphere is no low background but for the first prototype aiming at the measurement of resolution this is not indeed a key point, and the use of aluminium instead of copper made the fabrication process much cheaper. The setup was located at PRISNA facility [3] at CENBG assuring a running environment with a controlled temperature. In addition efforts were made to obtained vibrational insulation of tyhe supporting structure and custom made low noise electronics was used to reduce as much as possible the electronic noise (OWEN project [4]).

The setup was commissioned with $\mathrm{ArP} 2$ gas i.e. $98 \% \mathrm{Ar}$ and $2 \% \mathrm{CH}_{4}$ at pressures up to 1.1 bar. A new sphere certified to be operated at higher pressure up to 40 bar and a xenon recuperation system will be available soon for the first tests at high pressure with xenon expected in 2021. 


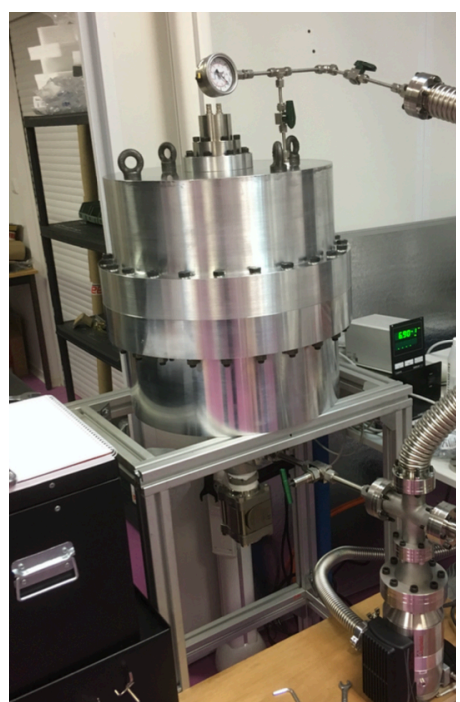

(a)

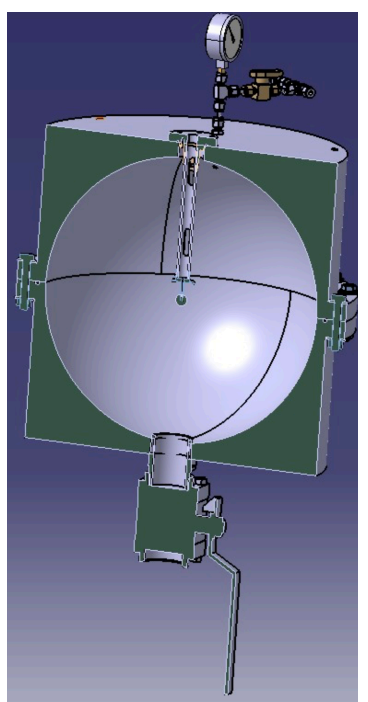

(b)

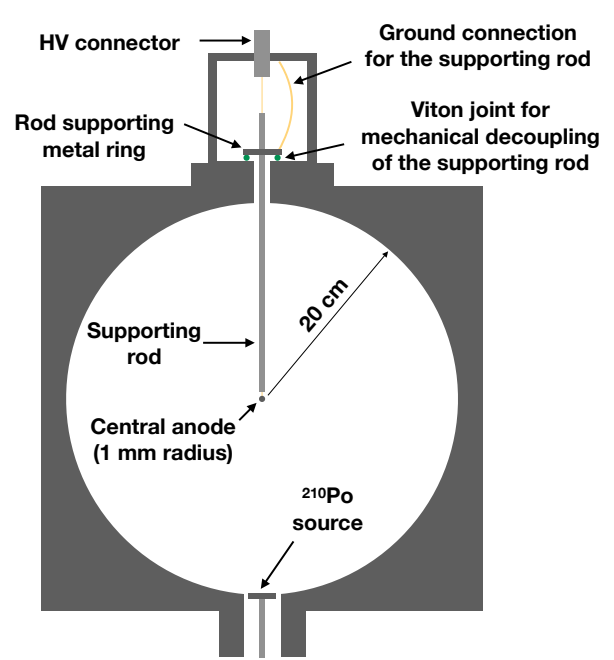

(c)

Figure 2: Actual realisation (a) and mechanical drawing (b) of the R2D2 detector. The two hemispheres can be seen as well as the pressure gauge, the HV connectors on top of the detector, and the large valve at the bottom to allow for the insertion of a radioactive source. (c) Schematic drawing of the experimental setup showing the central sensor support and the ${ }^{210}$ Po $\alpha$ source.

To assess the energy resolution a $4 \mathrm{~Bq}^{210} \mathrm{Po} \alpha$ source of $5.3 \mathrm{MeV}$ was used. The source deposited on a silver film is located on a support and inserted from the bottom of the detector as shown in Fig. 2(c).

\section{Results}

The first point to be tackled was to control the stability of the detector response in time. After obtaining a vacuum of $10^{-6} \mathrm{mbar}$ and measuring a leakage at the level of $5 \times 10^{-9} \mathrm{mbar} / \mathrm{s}$, the detector was filled with pure ArP2 gas. Several short runs of 30 minutes were taken over 14 days with the same gas, and during the run a pulse generator was used as input in the electronic chain to monitor possible electronics response variations.

A signal loss of $0.05 \%$ per hour was measured: this can be explained as due to electronegative impurities in the gas. Their concentration increases in time due to material outgassing or leakages and it results into a smaller number of electrons reaching the anode. The observed energy loss over time was expected and can be corrected offline, nonetheless to measure the energy resolution we performed short runs with new gas at each time so that we did not need to account for such an energy loss.

To assess energy resolution we took data at 1.1 bar with $\alpha$ tracks length of about $3-4 \mathrm{~cm}$, and 200 mbar where the mean length of $\alpha$ tracks is about $20 \mathrm{~cm}$. The waveforms were registered and several variables were computed to study the signal, in particular the total charge $Q t$ and the signal length $D t$. 


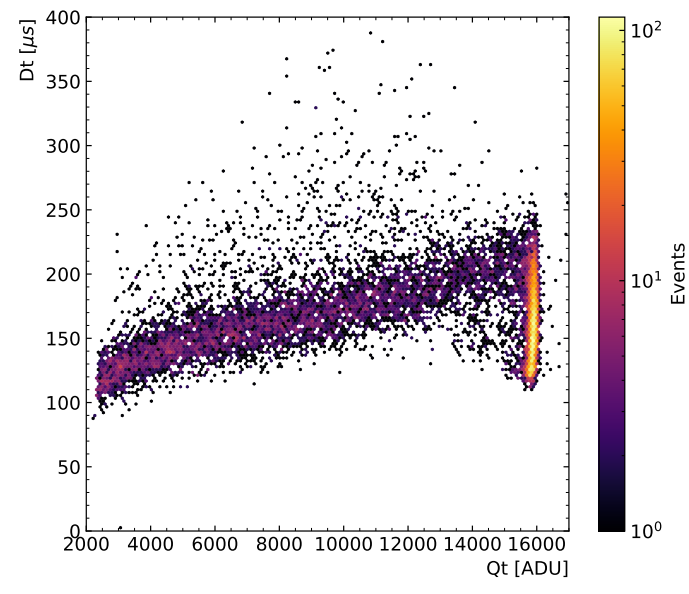

(a)

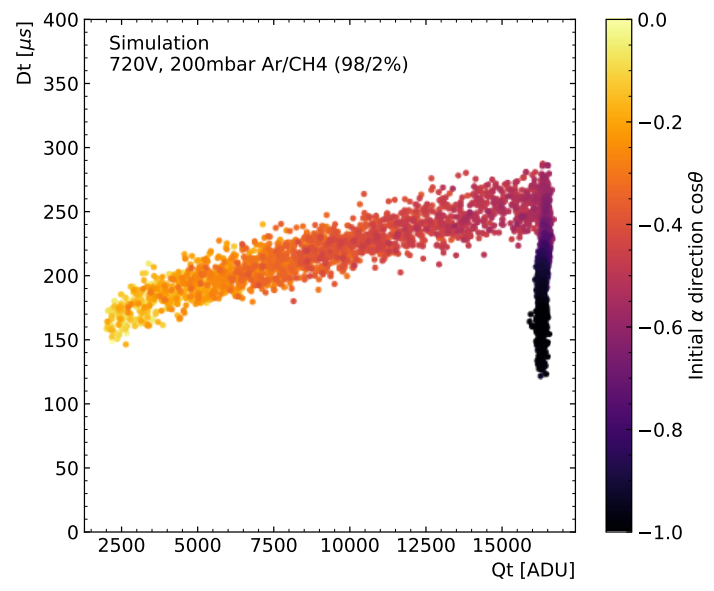

(b)

Figure 3: Example of 2D representation of events for different observables for data at 200 mbar and $720 \mathrm{~V}$ Dt vs Qt (a), and corresponding simulation (b). For the data the colour stands for the number of events in the bin whereas for the simulation the colour indicates the original $\alpha$ direction: $\cos \theta=-1$ for tracks going towards the central anode and $\cos \theta=0$ for tracks emitted orthogonally to the radial direction. The figures were made with about 14000 events without any selection cut, corresponding to about half an hour of data taking.

A dedicated simulation [5] based on Geant 4 and Garfield++ was used to cross check the obtained results and confirm our understanding of the detector response. The agreement between data and simulation is very good and the detector behaviour is well understood as can be seen in Fig 3. In particular the simulation allowed us to understand the shape of the waveforms as a function of the initial $\alpha$ direction to select different tracks topologies. A full analysis of the data can be found in Ref. [6].

The resolution was computed for the two data sets and we obtained a similar resolution of about $1.1 \%$ as can be seen in Fig. 4. The fact that the resolution does not change for the different pressures is very important since it demonstrates that the detector resolution does not depend on the length of the track.

\section{Next steps and conclusions}

The very promising results obtained so far have to be confirmed in xenon and at higher pressure. In order to do that a new detector certified for pressures up to 40 bar as well as a xenon recuperation system are needed: they will be delivered by the end of the year and results are expected in 2021. The results have been obtained with $\alpha$ tracks but we would like to measure the energy resolution with electrons: ${ }^{207} \mathrm{Bi}$ source is available, however we need to work at a pressure higher than $10 \mathrm{bar}$ to contain electrons tracks. Such a measurement is also foreseen in the second half of 2021.

Further R\&D is ongoing on the electronics development to reduce the detector noise, on the multi sensor anode (ACHINOS [7]) which could provide a coarse tracking and reduce the needed high voltage on the anode, and on the light readout. Indeed exploiting xenon scintillation light to have 


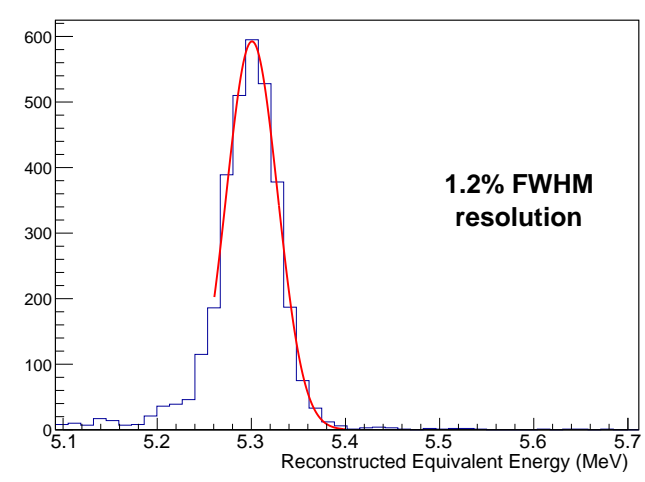

(a)

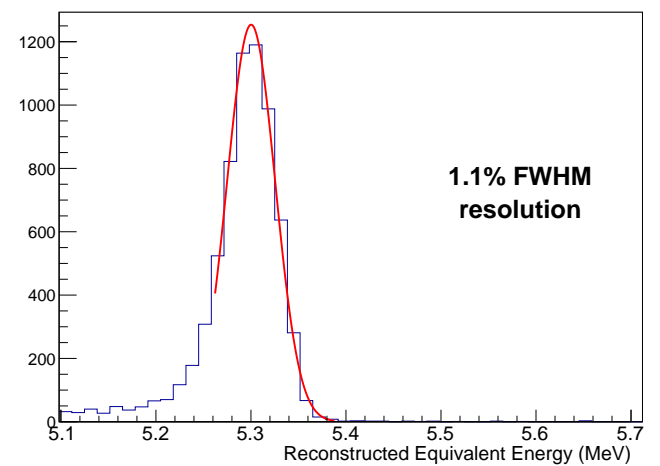

(b)

Figure 4: Integral of the $5.3 \mathrm{MeV} \alpha$ signal at 1.1 bar and $2000 \mathrm{~V}(\mathrm{a})$ and at $200 \mathrm{mbar}$ and $720 \mathrm{~V}(\mathrm{~b})$. The Gaussian fit in red shows an energy resolution of 1.2\% FWHM and 1.1\% FWHM respectively.

the starting time of the event would provide valuable information for the radial reconstruction of the energy deposits. Preliminary results using a silicon photomultiplier are very encouraging and we plan to do that in xenon soon.

To conclude the R2D2 project is an R\&D supported by IN2P3 to study the possibility of having a zero background ton scale detector for the search of $0 v \beta \beta$ decay exploiting a high pressure xenon TPC. A good detector understanding was demonstrated and a resolution at the level of $1.1 \%$ was achieved with alphas at $5.3 \mathrm{MeV}$. We also demonstrated that the energy resolution is not degraded going from point-like energy deposits to long particle tracks. Results have to be confirmed in xenon at higher pressure and depending on the success of the $R \& D$ we hope to move on in order to build a low background prototype allowing to provide the first physics results.

\section{References}

[1] Q. Arnaud et al. [NEWS-G Collaboration], Astropart. Phys. 97, 54 (2018).

[2] A. Meregaglia et al., JINST 13, no. 01, P01009 (2018).

[3] http://www.cenbg.in2p3.fr/-Plateforme-PRISNA-

[4] https://r2d2.in2p3.fr/owen.html

[5] I. Katsioulas et al., JINST 15, no.06, C06013 (2020).

[6] R. Bouet et al., [arXiv:2007.02570 [physics.ins-det]].

[7] A. Giganon et al., JINST 12, no.12, P12031 (2017) 